\title{
The correlation between patient reported outcomes and biomarkers for knee osteoarthritis
}

\author{
Bumsup Lee ${ }^{1}$, Martin Wang ${ }^{1}$, Soo-Hyun Lew ${ }^{2}$, Jung Jong Cho ${ }^{2}$, Tae Won Kim ${ }^{2}$, Nipun Sodhi ${ }^{3}$, \\ Hiba K. Anis ${ }^{4}$, Michael A. Mont ${ }^{3,4}$ \\ ${ }^{1}$ Kolon TissueGene, Inc., Rockville, MA, USA; ${ }^{2}$ Kolon Life science, Inc., Geumcheon-gu, Seoul, Korea; ${ }^{3}$ Department of Orthopaedic Surgery, Lenox \\ Hill Hospital, Northwell Health, New York, NY, USA; ${ }^{4}$ Department of Orthopaedic Surgery, Cleveland Clinic, Cleveland, OH, USA \\ Contributions: (I) Conception and design: All authors; (II) Administrative support: All authors; (III) Provision of study materials or patients: All \\ authors; (IV) Collection and assembly of data: All authors; (V) Data analysis and interpretation: All authors; (VI) Manuscript writing: All authors; \\ (VII) Final approval of manuscript: All authors. \\ Correspondence to: Michael A. Mont, MD. Department of Orthopaedic Surgery, Lenox Hill Hospital, Northwell Health, New York, NY, USA. \\ Email: mmont@northwell.edu; rhondamont@aol.com.
}

Background: Novel non-operative treatment modalities, including injections of genetically engineered chondrocytes (GEC), have been developed to target the inflammatory pathways implicated in knee osteoarthritis (OA). Patient reported outcome measures (PROMs) have been the cornerstone of knee OA diagnostics and are frequently used to quantify treatment efficacy but their subjective nature limits their utility. However, inflammatory biomarkers of knee OA provide quantitative data but are less commonly used to assess the severity and progression of OA. Therefore, the purpose of this study was to correlate PROMs with biomarkers in knee OA. Specifically, we compared International Knee Documentation (IKDC) scores with serum C-terminal telopeptide of type I collagen (sCTX-I), urine C-terminal telopeptide of type II collagen (uCTX-II), and joint space width (JSW) in patients receiving GEC injections or placebo injections for knee OA.

Methods: A multi-center, double-blinded, randomized, placebo-controlled study was conducted between November 2013 and August 2015. A total of 163 patients were enrolled and randomized to receive GEC injections or placebo injections in a 1:1 ratio of treatment to control. The PROMs assessed were IKDC scores which evaluated three domains: symptoms, sports and daily activity, and difference in knee function. The biomarkers assessed were sCTX-I, uCTX-II, and JSW. Outcomes were measured at baseline as well as at 6,9, and 12 months after the injections. Scatter plots were created to graphically represent the correlations. Pearson's correlation coefficients were utilized to determine associations between IKDC scores and each of the three biomarkers.

Results: Significant correlations were identified between PROMs and inflammatory biomarkers of knee $\mathrm{OA}$ at 6, 9, and 12 months post-treatment with GEC injections. In the GEC cohort, as IKDC scores improved, improvements in sCTX-I levels were also observed. Correlations between IKDC scores and sCTX-I levels were statistically significant at 9 months $(r=-0.42, \mathrm{P}<0.001)$ and 12 months $(r=-0.23, P=0.049)$ following treatment. Additionally, there were significant correlations between IKDC scores and uCTX-II levels in the GEC cohort at 6 months ( $\mathrm{r}=-0.294, \mathrm{P}=0.011), 9$ months $(\mathrm{r}=-0.383, \mathrm{P}<0.001)$, and 12 months $(\mathrm{r}=-0.420, \mathrm{P}<0.001)$ post-treatment. In both GEC and placebo cohorts, JSW was observed to improve as IKDC scores improved. In the GEC cohort, a statistically significant correlation was observed at 12 months post-treatment $(\mathrm{r}=0.400, \mathrm{P}<0.001)$.

Conclusions: Newer treatment options for knee OA target the inflammatory pathways that mediate the destruction of bone and cartilage. Inflammatory biomarkers are an objective alternative to assess the severity and progression of OA. This study found significant correlations between an established PROM (IKDC scores) and inflammatory biomarkers in knee OA (sCTX-I, uCTX-II, and JSW). For newer non-operative treatment modalities, such as GEC injections, inflammatory biomarkers of knee OA should be utilized to 
evaluate the severity and progression of knee OA.

Keywords: Osteoarthritis; biomarkers; patient reported outcomes; genetically engineered chondrocytes

Submitted Feb 08, 2019. Accepted for publication Feb 25, 2019.

doi: 10.21037/atm.2019.02.39

View this article at: http://dx.doi.org/10.21037/atm.2019.02.39

\section{Introduction}

Osteoarthritis (OA) is the most common joint disorder in the United States and knee OA in particular accounts for $80 \%$ of the total OA burden (1-5). Patient reported outcome measures (PROMs) have been the cornerstone of knee OA diagnostics and are frequently used to quantify treatment efficacy $(6,7)$. There are many variations of PROMs in knee OA including the International Knee Documentation (IKDC) Subjective Knee Evaluation Form, Western Ontario and McMaster Universities Osteoarthritis Index (WOMAC), and visual analog scale (VAS). The IKDC includes the scoring of three domains: symptoms (including pain, stiffness, instability, and swelling), sports and daily activity, and difference in knee function (8). The WOMAC consists of three slightly different subscales: severity of pain, severity of joint stiffness, and functional limitations. Lastly, VAS is a single scale item that measures pain on a continuous scale visualized on a $100 \mathrm{~mm}$ line and is commonly measured outcome (9). However, the subjective nature of all of these PROMs means that there may be high degrees of variation.

More recently, the inflammatory pathology in knee OA has been targeted in the development of new treatments which utilize the body's intrinsic abilities to reduce inflammation-mediated joint and cartilage damage. One such example is the development of genetically engineered allogenic chondrocytes (GEC). Results from phase I and II trials of this novel injection modality have been promising and have demonstrated substantial improvements in pain as well as function for knee OA patients. In such studies, several indicators of inflammation associated with knee OA can be evaluated including: serum C-terminal telopeptide of type I collagen (sCTX-I), urine C-terminal telopeptide of type II collagen (uCTX-II), and joint space width (JSW). Knee OA is characterized by deterioration of collagen and bone (10) and CTX biomarkers are indicative of collagen or bone degradation whereas JSW is commonly used to quantify OA progression.

Although PROMs are commonly utilized to evaluate the severity of knee OA, they are still limited by their subjectivity. Diagnoses of OA are primarily achieved with clinical and radiographic findings. However, with increasing literature on the use of inflammatory biomarkers (11-16), there is great potential for utilizing quantitative data to assess knee OA as well. Therefore, the purpose of this study was to correlate PROMs with inflammatory biomarkers in knee OA. Specifically, we compared IKDC scores with measurements of sCTX-I, uCTX-II, and JSW in patients receiving GEC injections for knee OA.

\section{Methods}

\section{Study population}

A double-blinded, randomized, placebo-controlled study was conducted between November 2013 and August 2015 to investigate the efficacy of GEC injections in treating knee OA. A total of 163 patients with knee OA were enrolled from twelve centers and followed up for 1 year. Institutional review board approval was obtained at all sites. Additional data from this phase III clinical trial has been previously published (17).

Patients were selected if they met the following inclusion criteria: diagnosis of Kellgren-Lawrence grade 3 radiographic knee OA, 19 years and older, body mass index (BMI) between 18.5 and $30 \mathrm{~kg} / \mathrm{m}^{2}$, and pain for longer than 3 consecutive months.

Patients were randomized to receive injections of either genetically engineered chondrocytes (GECs) virally transduced with TGF $\beta 1$ (GEC-TGF- $\beta 1$ ) (TissueGene-C; TissueGene Inc., Rockville, Maryland, USA) or a placebo ( $3 \mathrm{~mL}$ of $0.9 \%$ saline) in a $1: 1$ ratio of treatment to control. The GEC-TGF- $\beta 1$ cohort consisted of 80 patients and the control cohort consisted of 83 patients.

\section{Measured outcomes}

Outcomes were measured at baseline as well as at 6, 9, and 12 months after receiving injections. The PROMs 

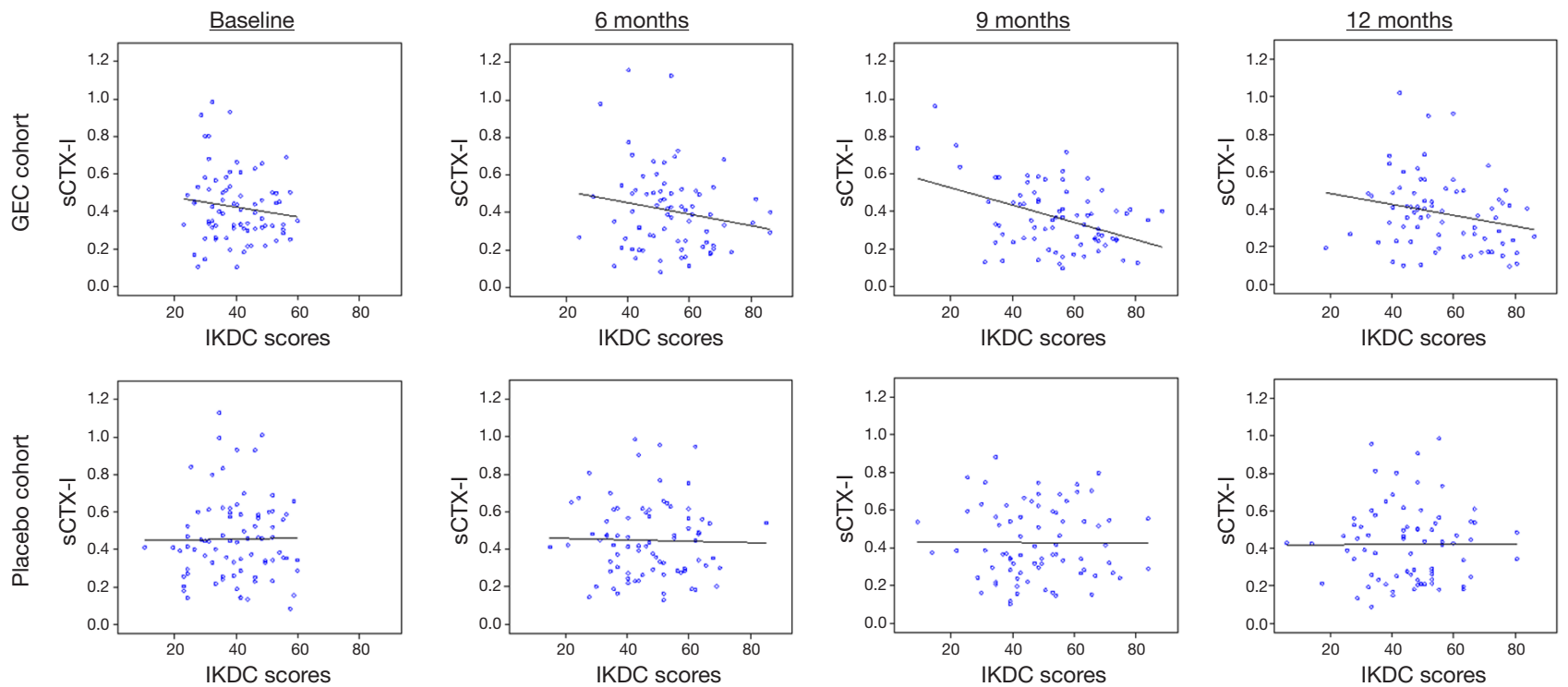

Figure 1 Scatter plots of IKDC scores and sCTX-I levels in the GEC cohort and placebo cohort. GEC, genetically engineered chondrocyte. sCTX-I, serum C-terminal telopeptide of type I collagen; IKDC, International Knee Documentation; JSW, joint space width; GEC, genetically engineered chondrocytes.

assessed were IKDC scores which evaluated three domains: symptoms, sports and daily activity, and difference in knee function. Responses are given through varied methods such as yes or no, 5-point Likert scales, or 0-10 numerical scales. A total score was calculated and higher scores corresponded to better outcomes (8).

The biomarkers assessed in this study were sCTX-I, uCTX-II, and JSW. Serum levels of CTX-I are indicative of the degradation of collagen type I found in bone (18) whereas CTX-II is indicative of the breakdown of collagen type II found in hyaline cartilage $(19,20)$. Articular cartilage loss can be indirectly inferred based on JSW whereby smaller values correspond to greater cartilage loss (21). JSW is the distance between the femoral condyle and tibial plateau and can be used to estimate structural disease progression with radiography (22). Currently, JSW is the accepted biomarker for measurement of $\mathrm{OA}$ progression in clinical trials by the European Agency for the Evaluation of Medicine Products and United States Food and Drug Administration (21).

\section{Data analyses}

Data from each patient were used to identify which of the biomarkers correlated with the PROMs studied. IKDC scores were compared to the biomarkers (sCTX-I, uCTXII, and JSW) and correlations were determined with linear regression. Scatter plots were created to graphically represent the correlations between IKDC scores and the biomarkers studied. Correlation analyses with Pearson's correlation coefficients (r) were utilized to identify positive or negative relationships between the outcome variables compared. Statistical significance was maintained at a $\mathrm{P}$ value of less than 0.05 .

\section{Results}

Significant correlations were identified between PROMs and inflammatory biomarkers of knee OA. Correlation analyses demonstrated significant associations between IKDC scores and the biomarkers at 6, 9, and 12 months post-treatment with GEC-TGF- $\beta 1$ injections.

In the GEC-TGF- $\beta 1$ cohort, as IKDC scores improved, improvements in sCTX-I levels were also observed (Figure 1). This was observed at baseline through to 12 months with negative correlations and demonstrated that as SCTX-I levels reduced (indicative of less degradation of collagen type I), IKDC scores increased (indicative of better outcomes). Of note, these correlations were statistically significant at 9 months $(\mathrm{r}=-0.42, \mathrm{P}<0.001)$ and 12 months $(\mathrm{r}=-0.23, \mathrm{P}=0.049)$ following treatment (Table 1).

Similar trends were observed between uCTX-II and IKDC scores (Figure 2). Negative correlations were 
Table 1 Correlations of biomarkers with IKDC scores

\begin{tabular}{|c|c|c|c|c|c|c|c|c|}
\hline Biomarker & \multicolumn{2}{|c|}{ Baseline } & \multicolumn{2}{|c|}{6 months } & \multicolumn{2}{|c|}{9 months } & \multicolumn{2}{|c|}{12 months } \\
\hline \multicolumn{9}{|l|}{ sCTX-I } \\
\hline GEC cohort & -0.133 & 0.242 & -0.179 & 0.126 & -0.424 & $<0.001$ & -0.229 & 0.0493 \\
\hline Placebo cohort & 0.014 & 0.900 & -0.027 & 0.817 & -0.008 & 0.945 & 0.401 & 0.9636 \\
\hline GEC cohort & -0.204 & 0.074 & -0.294 & 0.011 & -0.383 & $<0.001$ & -0.420 & $<0.001$ \\
\hline Placebo cohort & -0.114 & 0.311 & -0.110 & 0.339 & -0.029 & 0.803 & -0.084 & 0.469 \\
\hline \multicolumn{9}{|l|}{ JSW } \\
\hline GEC cohort & 0.186 & 0.109 & 0.267 & 0.2035 & & & 0.401 & $<0.001$ \\
\hline
\end{tabular}

r, Pearson's correlation coefficient. SCTX-I, serum C-terminal telopeptide of type I collagen; uCTX-II, urine C-terminal telopeptide of type II collagen; JSW, joint space width; GEC, genetically engineered chondrocytes.
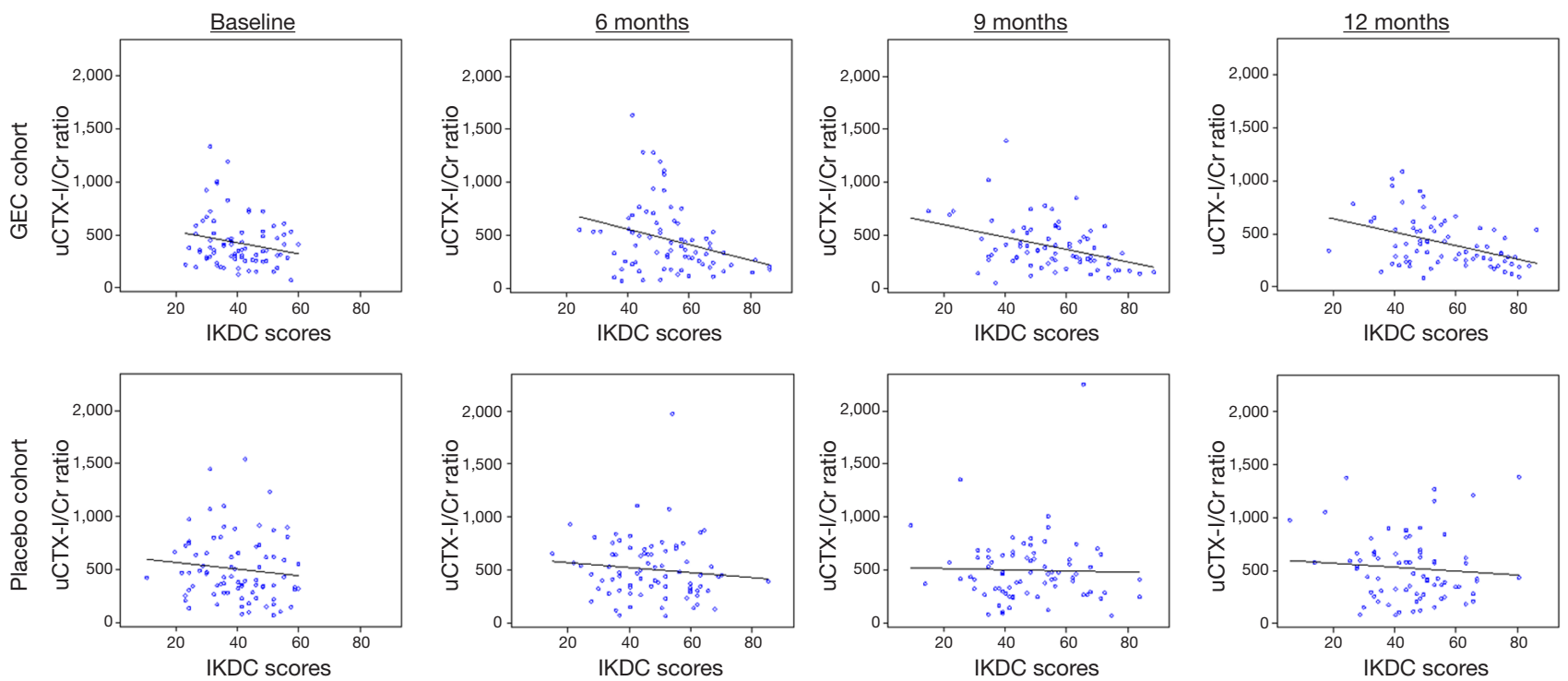

Figure 2 Scatter plots of IKDC scores and uCTX-II in the GEC cohort and placebo cohort. uCTX-II, urine C-terminal telopeptide of type II collagen; IKDC, International Knee Documentation; JSW, joint space width; GEC, genetically engineered chondrocytes.

observed between uCTX-II levels and IKDC scores from baseline to 12 months in both the placebo and GECTGF- $\beta 1$ cohorts. This demonstrated that lower levels of uCTX-II, and therefore hyaline cartilage breakdown, were associated with higher IKDC scores, and therefore improved PROMs. Additionally, these correlations were significant in the GEC-TGF- $\beta 1$ cohort at 6 months $(\mathrm{r}=-0.294, \mathrm{P}=0.011), 9$ months $(\mathrm{r}=-0.383, \mathrm{P}<0.001)$, and
12 months $(\mathrm{r}=-0.420, \mathrm{P}<0.001)$ post-treatment.

In both cohorts, JSW was observed to improve as IKDC scores improved (Figure 3). This was revealed with the positive correlations seen from baseline to 12 months after the injections; patients reported higher IKDC scores when JSW was greater. In the GEC-TGF- $\beta 1$ cohort, this correlation was statistically significant at 12 months after receiving treatment $(\mathrm{r}=0.400, \mathrm{P}<0.001)$. 

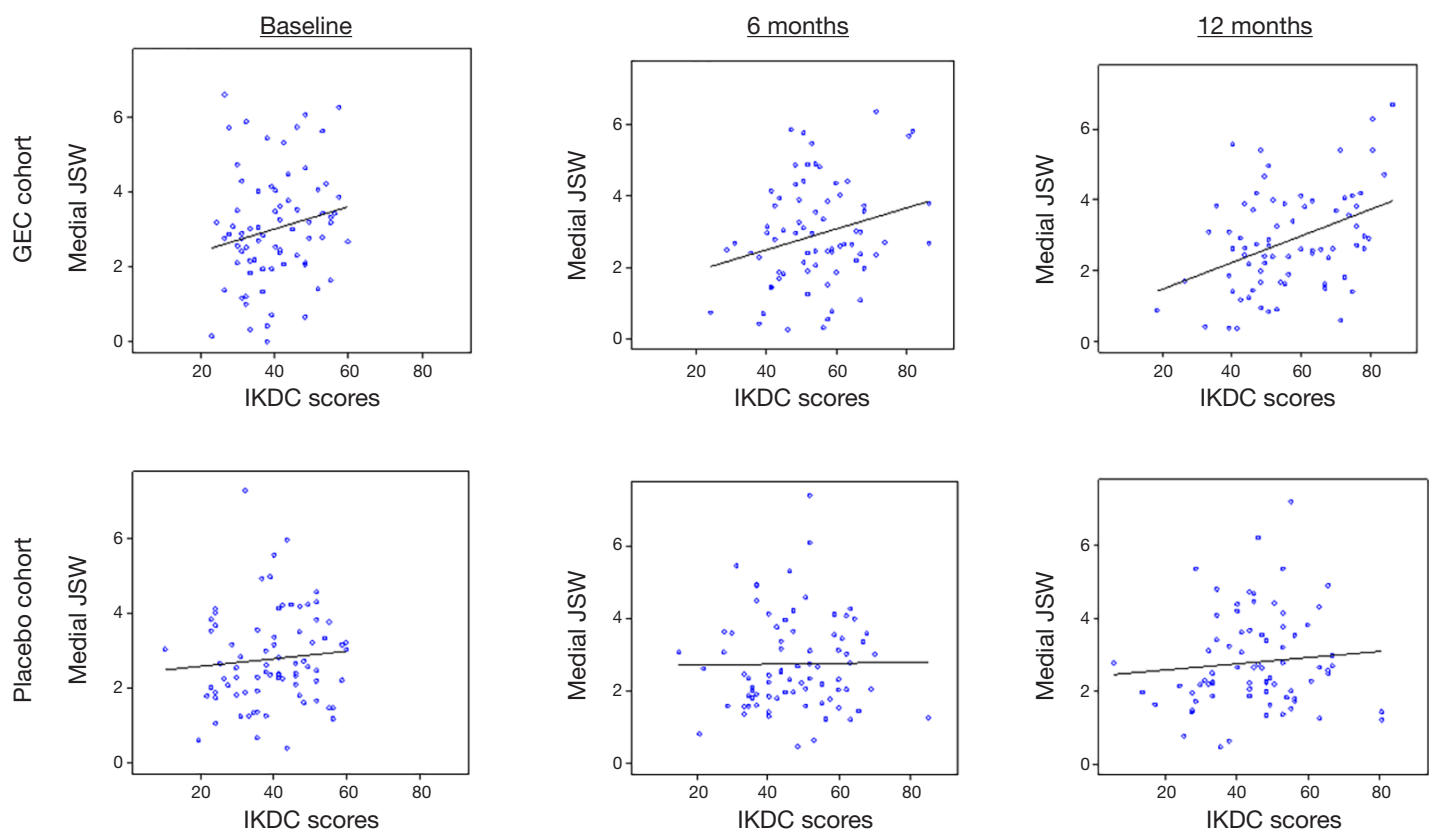

Figure 3 Scatter plots of IKDC scores and JSW in the GEC cohort and placebo cohort. IKDC, International Knee Documentation; JSW, joint space width; GEC, genetically engineered chondrocytes.

\section{Discussion}

Knee OA has long been recognized as an inflammatory disease, however, its diagnosis and progression are still heavily based on clinical and radiographic findings. There is a paucity of literature investigating the potential associations between inflammatory biomarkers of knee OA and commonly used PROMs. The purpose of this study was to determine correlations between markers of bone and cartilage degradation and patient-reported IKDC scores. Our results indicate that sCTX-I, uCTX-II, and JSW have strong correlations with PROMs. These findings build on existing literature demonstrating the important role of inflammatory pathology in the clinical progression of knee OA. Furthermore, this study reveals the great potential of novel GEC injections for the management of knee OA.

This study had some limitations. There is a need for longer term studies as the follow-up for the patients enrolled in the current study was limited to 12 months. Nevertheless, these preliminary results with early outcomes revealed correlations throughout the observed study period and therefore warrant future investigations on long-term outcomes. Additionally, the enrolled patients were not screened for undiagnosed hip OA, a potential secondary cause of pain which may influence the recorded PROMs. However, despite this limitation, this double-blinded, randomized, placebo-controlled study revealed biomarkers of knee OA were found to have significant correlations with PROMs at several time points in the study.

Similar to our findings, previous studies have also reported correlations between PROMs and inflammatory biomarkers (11-13). Mabey et al. (11) investigated the correlation of plasma and synovial autotaxin, a critical stimulator of inflammatory processes, with WOMAC scores and radiographic Kellgren-Lawrence grading in 70 patients with knee OA. The group found that plasma and synovial autotaxin levels had significant correlations with patientreported WOMAC scores $(\mathrm{r}=0.558, \mathrm{P}<0.001$ and $\mathrm{r}=0.371$, $\mathrm{P}=0.002$, respectively) and concluded that autotaxin levels have the potential to reflect the severity of knee OA. Dong et al. (12) also studied WOMAC scores and KellgrenLawrence scores but compared them to serum and synovial fluid levels of calcitonin gene-related peptide (CGRP). The group found that serum and synovial CGRP concentrations were positively correlated with Kellgren-Lawrence grades, total WOMAC scores, and each WOMAC subscale scores. These findings demonstrated that serum CGRP can be selected as a biomarker for monitoring and predicting the prognosis and progression of knee OA. Additionally, Das Gupta et al. (13) found correlations between inflammatory cytokines and WOMAC scores in 90 patients with knee 
OA. In this study, log serum IL-16 levels were significantly correlated with WOMAC scores $(\mathrm{P}=0.001)$ as well as the individual WOMAC subscale scores for pain $(\mathrm{P}=0.005)$, stiffness $(\mathrm{P}=0.019)$, and physical function $(\mathrm{P}<0.0001)$.

There is also substantial literature demonstrating the strong correlations between clinical severity of knee OA and levels of inflammatory biomarkers (14-16). In a study of 89 patients with knee OA, Botha-Scheepers et al. (15) found that after adjusting for age, sex, and BMI, the progression of joint space narrowing (JSN) was 6 times higher for patients in the highest quartile of TNF-alpha production (RR 6.1; $95 \%$ CI, 1.4 to 9.8 ) and 4 times higher for patients in the highest quartile of IL-10 production (RR 4.3; 95\% CI, 1.7 to 6.2) compared to the lowest respective quartiles. Similarly, Daghestani et al. (14) studied 184 patients with knee OA to evaluate activated macrophage markers, CD14 and CD163, and their associations with osteophyte progression, JSN and joint pain. After adjusting for age, sex, and BMI, the group found that levels of CD14 in both synovial fluid and plasma were positively associated with self-reported knee pain ( $\beta=0.773, \mathrm{P}=0.003$ and $\beta=0.641, \mathrm{P}=0.031$, respectively). Synovial fluid levels of CD14 and CD163 were also positively associated with osteophyte progression $(\beta=0.096, \mathrm{P}=0.015$ and $\beta=0.072, P=0.007$, respectively).

In contrast, there are studies that have shown certain inflammatory biomarkers are not associated with clinical outcomes in knee OA $(23,24)$. Mahler et al. (23) performed a study looking at S100A8/S100A9 serum levels with clinical and structural characteristics with hip, knee, and hand OA in 162 patients. The group found that serum S100A8/A9 did not have any associations with WOMAC scores $(\mathrm{P}<0.05)$ and concluded that serum S100A8/A9 was not an acceptable biomarker for clinical OA. However, it is important to note that unlike the biomarkers included in our current study, S100A8/ A9 is not indicative of collagen damage or degradation. Moreover, the study by Mahler et al. analyzed combined data from hip, knee, and hand OA and which limits its interpretation for knee OA alone. Another study performed by Leung et al. (24) evaluated the relationships of pro-inflammatory biomarkers and matrix degradation biomarkers with knee pain and WOMAC scores in 109 patients with knee OA. The group found no significant associations between matrix degradations markers and WOMAC scores. However, after adjusting for age, sex, and BMI, synovial fluid TNF-alpha was found to have significant associations with WOMAC total pain scores $(\beta=34.61,95 \%$ CI, 6.01 to $63.21, \mathrm{P}<0.05)$.

\section{Conclusions}

Novel treatment modalities for knee OA target the inflammatory pathways that mediate the destruction of bone and cartilage. Injections of GEC is one such treatment which is currently being investigated and has shown promising results in phase I and II trials. Although PROMs are an accepted form of evaluation when assessing management strategies for knee OA, they are largely subjective and are therefore variably reported between patients. Biomarkers are a more objective alternative and provide quantitative data that can be used to assess the severity and progression of OA. This study found significant correlations between an established PROM (IKDC scores) and inflammatory biomarkers in knee OA (sCTX-I, uCTX-II, and JSW). For newer non-operative treatment modalities such as GEC injections, inflammatory biomarkers of knee OA should be utilized to evaluate the severity and progression of knee OA.

\section{Acknowledgements}

None.

\section{Footnote}

Conflicts of Interest: B Lee: TissueGene; M Wang: TissueGene; SH Lew: Life Science; JJ Cho: Life Science; TW Kim: Life Science; MA Mont: AAOS, Cymedica, DJ Orthopaedics, Johnson \& Johnson, Journal of Arthroplasty, Journal of Knee Surgery, Microport, National Institutes of Health (NIAMS \& NICHD), Ongoing Care Solutions, Orthopedics, Orthosensor, Pacira, Peerwell, Performance Dynamics Inc, Sage, Stryker: IP royalties, Surgical Technologies International, Kolon TissueGene. The other authors have no conflicts of interest to declare.

Ethical Statement: Institution Review Board approval was obtained prior to the initiation of this study.

\section{References}

1. Felson DT, Lawrence RC, Dieppe PA, et al. Osteoarthritis: new insights. Part 1: the disease and its risk factors. Ann Intern Med 2000;133:635-46.

2. Lawrence RC, Felson DT, Helmick CG, et al. Estimates of the prevalence of arthritis and other rheumatic conditions in the United States: Part II. Arthritis Rheum 2008;58:26-35. 
3. Vos T, Flaxman AD, Naghavi M, et al. Years lived with disability (YLDs) for 1160 sequelae of 289 diseases and injuries 1990-2010: a systematic analysis for the Global Burden of Disease Study 2010. Lancet 2012;380:2163-96.

4. Zhang Y, Jordan JM. Epidemiology of Osteoarthritis. Clin Geriatr Med 2010;26:355-69.

5. Felson DT. Osteoarthritis of the Knee. N Engl J Med 2006;354:841-8.

6. Ramkumar PN, Harris JD, Noble PC. Patient-reported outcome measures after total knee arthroplasty: a systematic review. Bone Joint Res 2015;4:120-7.

7. Bourne RB. Measuring tools for functional outcomes in total knee arthroplasty. Clin Orthop Relat Res 2008;466:2634-8.

8. Collins NJ, Misra D, Felson DT, et al. Measures of knee function: International Knee Documentation Committee (IKDC) Subjective Knee Evaluation Form, Knee Injury and Osteoarthritis Outcome Score (KOOS), Knee Injury and Osteoarthritis Outcome Score Physical Function Short Form (KOOS-PS), Knee Outcome Survey Activities of Daily Living Scale (KOS-ADL), Lysholm Knee Scoring Scale, Oxford Knee Score (OKS), Western Ontario and McMaster Universities Osteoarthritis Index (WOMAC), Activity Rating Scale (ARS), and Tegner Activity Score (TAS). Arthritis Care Res (Hoboken) 2011;63 Suppl 1:S208-28.

9. Bodian CA, Freedman G, Hossain S, et al. The visual analog scale for pain: clinical significance in postoperative patients. Anesthesiology 2001;95:1356-61.

10. Heidari B. Knee osteoarthritis diagnosis, treatment and associated factors of progression: part II. Caspian J Intern Med 2011;2:249-55.

11. Mabey T, Taleongpong $\mathrm{P}, \mathrm{Udom}$ inprasert $\mathrm{W}$, et al. Plasma and synovial fluid autotaxin correlate with severity in knee osteoarthritis. Clin Chim Acta 2015;444:72-7.

12. Dong T, Chang H, Zhang F, et al. Calcitonin generelated peptide can be selected as a predictive biomarker on progression and prognosis of knee osteoarthritis. Int Orthop 2015;39:1237-43.

13. Das Gupta E, Ng WR, Wong SF, et al. Correlation of serum cartilage oligomeric matrix protein (COMP) and interleukin-16 (IL-16) levels with disease severity in primary knee osteoarthritis: A pilot study in a Malaysian population. PLoS One 2017;12:e0184802.

14. Daghestani HN, Pieper CF, Kraus VB. Soluble macrophage biomarkers indicate inflammatory phenotypes in patients with knee osteoarthritis. Arthritis Rheumatol 2015;67:956-65.

15. Botha-Scheepers S, Watt I, Slagboom E, et al. Innate production of tumour necrosis factor and interleukin 10 is associated with radiological progression of knee osteoarthritis. Ann Rheum Dis 2008;67:1165-9.

16. Benito MJ, Veale DJ, FitzGerald O, et al. Synovial tissue inflammation in early and late osteoarthritis. Ann Rheum Dis 2005;64:1263-7.

17. Kim MK, Ha CW, In Y, et al. A Multicenter, Double-Blind, Phase III Clinical Trial to Evaluate the Efficacy and Safety of a Cell and Gene Therapy in Knee Osteoarthritis Patients. Hum Gene Ther Clin Dev 2018. [Epub ahead of print].

18. Vos LM, Kuijer R, Huddleston Slater JJ, et al. Inflammation Is More Distinct in Temporomandibular Joint Osteoarthritis Compared to the Knee Joint. J Oral Maxillofac Surg 2014;72:35-40.

19. Oestergaard S, Chouinard L, Doyle N, et al. The utility of measuring C-terminal telopeptides of collagen type II (CTX-II) in serum and synovial fluid samples for estimation of articular cartilage status in experimental models of destructive joint diseases. Osteoarthr Cartil 2006;14:670-9.

20. Lohmander LS, Atley LM, Pietka TA, et al. The release of crosslinked peptides from type II collagen into human synovial fluid is increased soon after joint injury and in osteoarthritis. Arthritis Rheum 2003;48:3130-9.

21. Hunter DJ, Le Graverand MP, Eckstein F. Radiologic markers of osteoarthritis progression. Curr Opin Rheumatol 2009;21:110-7.

22. Oak SR, Ghodadra A, Winalski CS, et al. Radiographic joint space width is correlated with 4-year clinical outcomes in patients with knee osteoarthritis: data from the osteoarthritis initiative. Osteoarthr Cartil 2013;21:1185-90.

23. Mahler EA, Zweers M, van Lent P, et al. Association between serum levels of the proinflammatory protein S100A8/A9 and clinical and structural characteristics of patients with established knee, hip, and hand osteoarthritis. Scand J Rheumatol 2015;44:56-60.

24. Leung YY, Huebner JL, Haaland B, et al. Synovial fluid pro-inflammatory profile differs according to the characteristics of knee pain. Osteoarthr Cartil 2017;25:1420-7.

Cite this article as: Lee B, Wang M, Lew SH, Cho JJ, Kim TW, Sodhi N, Anis HK, Mont MA. The correlation between patient reported outcomes and biomarkers for knee osteoarthritis. Ann Transl Med 2019. doi: 10.21037/ atm.2019.02.39 